\title{
Evaluation of multiple variate selection methods from a biological perspective: a nutrigenomics case study
}

\author{
Henri S. Tapp • Marijana Radonjic • \\ E. Kate Kemsley $\cdot$ Uwe Thissen
}

Received: 13 October 2011/ Accepted: 8 February 2012/Published online: 2 March 2012

(c) The Author(s) 2012. This article is published with open access at Springerlink.com

\begin{abstract}
Genomics-based technologies produce large amounts of data. To interpret the results and identify the most important variates related to phenotypes of interest, various multivariate regression and variate selection methods are used. Although inspected for statistical performance, the relevance of multivariate models in interpreting biological data sets often remains elusive. We compare various multivariate regression and variate selection methods applied to a nutrigenomics data set in terms of performance, utility and biological interpretability. The studied data set comprised hepatic transcriptome (10,072 predictor variates) and plasma protein concentrations $[2$ dependent variates: Leptin (LEP) and Tissue inhibitor of metalloproteinase 1 (TIMP-1)] collected during a high-fat diet study in
\end{abstract}

Henri S. Tapp and Marijana Radonjic contributed equally to this work.

Electronic supplementary material The online version of this article (doi:10.1007/s12263-012-0288-4) contains supplementary material, which is available to authorized users.

H. S. Tapp · E. Kate Kemsley

Institute of Food Research, Norwich Research Park,

Colney Lane, Norwich NR4 7UA, UK

M. Radonjic ( $\square)$

TNO, Microbiology and Systems Biology, P.O. Box 360,

3700 AJ Zeist, The Netherlands

e-mail: marijana.radonjic@tno.nl

M. Radonjic · U. Thissen

Nutrigenomics Consortium, Top Institute Food and Nutrition,

P.O. Box 557, 6700 AN Wageningen, The Netherlands

Present Address:

U. Thissen

Keygene N.V., P.O. Box 216, 6700 AE Wageningen,

The Netherlands
ApoE3Leiden mice. The multivariate regression methods used were: partial least squares "PLS"; a genetic algorithmbased multiple linear regression, "GA-MLR"; two leastangle shrinkage methods, "LASSO" and "ELASTIC NET"; and a variant of PLS that uses covariance-based variate selection, "CovProc." Two methods of ranking the genes for Gene Set Enrichment Analysis (GSEA) were also investigated: either by their correlation with the protein data or by the stability of the PLS regression coefficients. The regression methods performed similarly, with CovProc and GA performing the best and worst, respectively ( $R$-squared values based on "double cross-validation" predictions of 0.762 and 0.451 for LEP; and 0.701 and 0.482 for TIMP-1). CovProc, LASSO and ELASTIC NET all produced parsimonious regression models and consistently identified small subsets of variates, with high commonality between the methods. Comparison of the gene ranking approaches found a high degree of agreement, with PLS-based ranking finding fewer significant gene sets. We recommend the use of CovProc for variate selection, in tandem with univariate methods, and the use of correlationbased ranking for GSEA-like pathway analysis methods.

Keywords Multivariate statistical analysis .

Nutrigenomics - Microarrays · Pathway analysis .

High-fat diet

\section{Introduction}

In many life science studies, large data sets are generated from metabolomics, proteomics and transcriptomics experiments. Measurement of numerous relevant metabolites, proteins and genes in a single experiment allows an almost unbiased investigation into the important potential 
biomarkers or crucial pathways associated with the original study goal. Interpreting the results, however, requires dedicated techniques that can select or rank variates from large amounts of data. Usually, statistical models are generated that describe the relationship between the genomics data and some feature of interest (e.g., a phenotype). The models are then further analyzed to identify the most important variates.

Many variate selection methods are described in the literature. These can differ in their implementation details or in their fundamental statistical principles (Guyon and Elisseeff 2003; Guyon et al. 2006). An ideal variate selection method has principles and parameters that are well-suited to the particular study goal and/or to the data characteristics, although it is not always straightforward to make these choices in advance. Furthermore, even though the statistical principles of a method may be understood, its utility from a biological perspective is often less obvious.

This paper describes the performance of a number of variate selection or ranking techniques, from both a statistical and biological perspective. Representative of quite dissimilar approaches, the statistical methods used are:

- Partial least squares (PLS) regression (Martens and Naes 1989) — a latent vector (LV) approach;

- Genetic algorithm (GA) (Mitchelle 1998)—a global optimization approach, combined with multiple linear regression (MLR);

- LASSO (Tibshirani 1996) and ELASTIC NET (Zou and Hastie 2005)_least-angle shrinkage approaches;

- Covariance procedure (CovProc) - a PLS variant that uses variate selection based on covariance (Reinikainen and Höskuldsson 2003).

In broad terms, these all involve multivariate regression modeling of some kind and the estimation of a few "meta parameters" to summarize the model complexity. We have additionally made comparisons with univariate regression models built from individual genes and the reference protein data.

The methods were applied to quantitative protein measurements and microarray gene expression data obtained from a nutrigenomics case study described in Radonjic et al. (2009). Nutrigenomics investigates molecular relationships between dietary components and genes, proteins and/or metabolites on a large scale. It addresses the question of how nutrition influences gene transcription, protein expression and/or metabolism, with the aim of understanding how dietary factors can affect an individual's health on a systems level (Müller and Kersten 2003; Afman and Müller 2006; Baccini et al. 2008; Kaput et al. 2010; Evelo et al. 2011). The data used in the present work originate from a large-scale nutritional intervention survey performed in Apolipoprotein E3-Leiden (ApoE3Leiden) transgenic mice (Radonjic et al. 2009). The study examined the time-resolved development of high-fat-induced obesity and related pathologies and used microarrays to obtain genome-wide hepatic gene expression data. These have been used as the predictors in the variate selection methods. We have focused on this single data set to allow a detailed evaluation of the biological relevance of the genes and gene sets selected by the statistical approaches used in this study. Two dependent variates have been considered: plasma concentrations of the proteins Leptin (LEP) and Tissue inhibitor of metalloproteinase 1 (TIMP-1). They were chosen for their relevance to obesity development and inflammation-related tissue remodeling upon high-fat feeding, respectively.

\section{Materials and methods}

Study design, tissue collection and analysis

A detailed description of the study characteristics including study design, sample preparation, RNA isolation and quality control is described by Radonjic et al. (2009). This section only describes the parts that are relevant for understanding the present work.

The study involved a longitudinal comparison of hepatic gene expression between animals fed a control diet and those fed diets high in either animal or vegetable fat. The mRNA expression levels were determined using NuGO Affymetrix Mouse GeneChip arrays (NuGO_Mm1a 520177) and hepatic RNA material from groups of animals from each diet immolated at eight time points ( 0 days (chow fed), 1 day, 3 days, 1, 2, 4, 8, 12 and 16 weeks) during a 16-week trial. In total, 116 microarray samples were taken for further analysis, comprising 3-6 biological replicates per diet, per time-point. After applying data preprocessing pipeline, hepatic gene expression values were obtained for 15,105 genes with unique identifiers and 73 Affymetrix controls. From a total of 15,178 features, 10,072 were selected based upon the following two criteria: first, for at least one of the diet-time categories, there were two or more absolute expression values greater than a threshold value of 5 units. Second, the maximum-to-minimum expression ratio was $>1.5$, equivalent to a difference of 0.585 in $\log _{2}$ transformed data. Such expression data set has been used as the predictors in the variate selection methods.

In the same high-fat feeding study, plasma concentrations of multiple inflammatory proteins and chemokines were measured with multiplex technologies (Rodent Map v.2.0, Rules Based Medicine, USA). In total, protein data were available for 115 animals. Two proteins (LEP and TIMP-1) were considered as dependant variables for 
assessing the performance of the variate selection methods evaluated in the current study.

In total, 88 ApoE3Leiden liver and plasma samples were selected from the original study, on the basis of animalmatching data availability for both hepatic transcriptomics and protein measurements for assessing the performance of variate selection methods in the current study. The size of the gene expression matrix analyzed in this study was, therefore, of dimensions [ $88 \times 10,072]$. The matching 88 animals included 31 mice fed chow diet, 33 mice fed animal fat diet and 24 mice fed plant fat diet.

\section{Regression analysis}

Multivariate modeling and univariate correlation analysis were performed using Matlab (Mathworks Inc.). The Matlab modeling routines are available on request. The transcriptomics data were $\log _{2}$ transformed before analysis, which is a standard step prior to statistical analysis (Van den Berg 2006). All the regression methods used unit variance scaling. Models were assessed by cross-validation using 10 blocks. Single cross-validation (SCV) was used to determine the final model's meta-parameters, and double cross-validation (DCV) (Smit et al. 2007; Stone 1974) was used to assess predictive performance and model consistency.

Partial Least Squares (PLS) Partial least squares is a well-known supervised multivariate latent vector modeling technique (Boulesteix and Strimmer 2007; Martens and Naes 1989). It is not a variate selection method. The number of PLS factors that minimized a modified form of the Amemiya Prediction Criterion, APC, (Norušis and SPSS Inc 1990) was considered to be the optimal metaparameter: $\operatorname{APC}(a)=[(n+a) /(n-a)]\left[1-\left(r_{\mathrm{scv}}\right)^{2}\right]$, where $n$ is the number of observations, $a$ is the number of PLS factors used in the model, and $r_{\mathrm{scv}}$ is the Pearson correlation between the actual values and single cross-validated predictions. The stability of the regression coefficient was calculated by dividing the final SCV coefficients by a jackknife estimate of their standard error, calculated from both DCV and SCV results, as described by Faber (2002). This was used as a basis for ranking the genes for use in conjunction with Gene Set Enrichment Analysis (see below).

Genetic Algorithm (GA) Genetic algorithm in combination with multiple linear regression (MLR) was implemented according to Kemsley et al. (2007) and McLeod et al. (2009). The GA is a global optimization variate selection method that builds MLR models based on the best subset of variates. The closest analogue to a meta-parameter is the number of variates used in the final model. GA regression was implemented using an in-house scheme developed at the Institute of Food Research. The GA is a global optimization variate selection method that builds multiple linear regression models based on small subsets of variates. The GA aims to optimize both the model size (number of variates) as well as identifying the best subset. The minimum model size considered was 2 variates, and the maximum size was 69 and 78 for double cross-validation, $\mathrm{DCV}$, and single cross-validation, $\mathrm{SCV}$, respectively. Population sizes of 340 and 308 models were used for the DCV and SCV, respectively. The model fitness criterion used was the mean squared residuals based on block cross-validation. The cross-validation partitions were permuted after each generation. The most successful (fittest) model automatically passed to the next generation. All models in the current population could potentially act as parents although the breading probability was weighted toward the fitter models. The algorithm halts if either of two criteria is met: 30 generations without a change in the fittest model, or if a maximum of $\sim 200$ generations has passed. The size of the offspring model is chosen as a randomly assigned number that spans the size range of the parents, with a finite possibility of this value reducing by one. There are three mutation mechanisms: in neighbor and correlation-based annealing, there are finite probabilities of one variate swapping with either an adjacent variate or with one of its five most correlated alternatives. The third mechanism is the possibility of replacing or including a new variate chosen from either the list of all possible variates or from those present in the current population. Duplicate progeny is replaced with immigrants with the same number of variates as the current best model.

Least absolute shrinkage and selection operator (LASSO) (Tibshirani 1996) finds regression coefficients that minimize the squared residuals while also being constrained such that the sum of the absolute coefficient values is less than a given value, $t$, which is the meta-parameter. The L1 constraint causes many of the regression coefficients to be set to zero, which makes LASSO a variate selection method. No upper limit was set to the number of variates used in candidate models and the optimum model chosen is that which minimized the Aikake Information Criteria, AIC (Norušis and SPSS Inc 1990).

Elastic Net is an extension to LASSO that uses an additional L2 “ridge-regression" constraint, $\lambda_{2}$, which is the second meta-parameter to be estimated (Zou and Hastie 2005). This overcomes two limitations of LASSO: (1) the number of selected variates in the model is restricted by the data sample size, and (2) only one variate is selected from a group of highly correlated ones. Candidate models were limited to a maximum of 200 variates.

Covariance procedure (CovProc) is a PLS-based variate selection method (Reinikainen and Höskuldsson 2003). The variates are ranked in descending order of the absolute magnitude of the coefficients of the first vector. For 
variance scaled data, this corresponds to introducing variates based on the strength of correlation with the dependant variate. Regression models were evaluated that used increasing numbers of variates, introduced in five-variate increments, based on the order of the ranked list. The values of the two model meta-parameters (number of variates, number of PLS factors) chosen in the final model corresponded to the combination that resulted in the overall minimum APC.

Biological interpretation of variate selection results

Ingenuity Pathway Analysis suite (IPA, Ingenuity ${ }^{\circledR}$ Systems. http://www.ingenuity.com, version date May 2009) was used to analyze biological functions of the genes in the final models of CovProc, LASSO, ELASTIC NET and GA, by employing "Overrepresentation analysis" module. Biological function overrepresentation analysis aims to gain a mechanistic insight into the underlying biology of a selected group of genes. It evaluates whether gene sets associated with particular biological functions-such as those represented by Gene Ontology (GO) annotationsare statistically overrepresented in the identified gene group (for example, list of differentially expressed genes or group of genes selected by multivariate models). In this study, Fisher's exact test $p$ values were used to score the significance of biological functions among the genes in the final models of the four appraised variate selection approaches.

Gene Set Enrichment Analysis (GSEA, Subramanian et al. 2005) was used to evaluate the biological relevance of ranking the genes based on two approaches: by their correlation $r$ with the protein data and by the stability of the PLS regression coefficients. The ranked gene lists were supplied as inputs into the PreRanked scoring procedure available within the GSEA software. In GSEA, a score is produced, similar to the Kolmogorov-Smirnov statistic, which summarizes the distribution of a predefined set of genes within a prioritized list of genes. Higher scores are given to gene sets that are distributed near the top or bottom of the list. The likelihood of achieving a given score is evaluated by recalculating the score after repeated random permutations of the list order. When multiple gene sets are evaluated, the permutation-based $p$ values are used to control the false discovery rate (FDR). Our analysis used gene sets from Molecular signature database (MSigDB) C2 curated gene sets collection (http://www.broad.mit.edu/ gsea/msigdb September 2008). A gene set size filter $(\min =15, \max =500)$ removed 737 of the 1,687 gene sets, leaving 950 to be used in the analysis. After collapsing 10,072 native features (gene identifiers from the gene expression data set) into gene symbols, 9,985 genes were recognized and used for the analysis. The number of permutations was set to 1,000 . The permutations are used to assign $p$ values to the GSEA scores calculated for each gene set. This avoids assuming the scores belong to some underlying distribution. As we evaluated 950 gene sets, the permutation-based $p$ values are also used to control the false discovery rate, FDR (e.g., Benjamini and Hochberg 1995). The significantly enriched gene sets referred to in the "Results and discussion" section are those that passed Benjamini and Hochberg FDR threshold: gene sets are considered significantly enriched at false discovery rate (FDR) smaller than $1 \%$ ( $q$ value $\leq 0.01)$.

The MSigDB service was used to find significant ( $p \leq 0.01$ ) overlaps between CovProc selected genes and gene sets in the collection.

\section{Results and discussion}

Hepatic genome-wide gene expression levels and plasma protein levels in high-fat diet fed ApoE3Leiden mice were analyzed using five multivariate regression methods: CovProc, LASSO, ELASTIC NET, GA and PLS ("Materials and methods"). The multivariate models were used to prioritize genes that predict the expression of two proteins associated with obesity phenotypes upon high-fat feeding, namely Leptin (LEP) and Tissue inhibitor of metalloproteinase 1 (TIMP-1). This allows elucidation of hepatic molecular mechanisms and the identification of biomarkers associated with deregulated adiposity and tissue remodeling, respectively, observed upon administration of high-fat diets.

Performance of five multivariate regression methods: model performance

The results of the double cross-validation (DCV) prediction comparison are shown in Table 1. For both proteins studied, CovProc and GA produced the best and worst

Table 1 Performance of five multivariate regression methods for the prediction of LEP and TIMP-1: predictions using double crossvalidation

\begin{tabular}{llllll}
\hline Method & \multicolumn{2}{l}{ LEP } & & \multicolumn{2}{l}{ TIMP-1 } \\
\cline { 2 - 3 } \cline { 6 - 7 } & $r^{2}$ & SEV & & $r^{2}$ & SEV \\
\hline LASSO & 0.614 & 1.65 & & 0.698 & 0.980 \\
ELASTIC NET & 0.577 & 1.75 & & 0.682 & 0.899 \\
CovProc & $\mathbf{0 . 7 6 2}$ & 1.31 & $\mathbf{0 . 7 0 1}$ & 0.864 \\
GA & 0.451 & 2.34 & & 0.482 & 1.242 \\
PLS & 0.621 & 1.63 & & 0.650 & 0.925 \\
\hline
\end{tabular}

Bold values indicate the best performance

$r^{2}$, squared correlation between predicted and actual values; SEV, root mean squared residuals 
predictions, respectively, CovProc only slightly exceeding LASSO, and all the variate selection methods performing comparably to PLS. These results can be put into context by considering the correlation $r$ between individual genes and the protein data. We find that the numbers of significantly $(p(r) \leq 0.05 / 10,072)$ correlated genes were 208 (2.1\% of all genes) and 486 (4.8\%) for LEP and TIMP-1, respectively. Single gene regression models were evaluated using single cross-validation (SCV) to allow direct comparison with the results in Table 1. For LEP and TIMP-1, respectively, 18 and 40 genes had an individual predictive ability greater than that obtained by GA; and for TIMP-1, one gene (Serpina3n) performed even better than CovProc. This is perhaps a surprising finding, as the widespread use of multivariate analysis (MVA) methods in traditional applications involving high-dimensional data, such as spectroscopy, is due to the improved predictive ability they offer through the "multivariate advantage," which deals with confounding systematic variation in the predictor data. Our findings imply that - for transcriptomic data, at leastunivariate methods should also be investigated.

Note that all the variate selection methods could potentially have selected a single variate, and in the case of TIMP-1, this would have led to an improved predictive performance. That all the MVA methods instead selected multiple variates can be interpreted as evidence of overfitting during the model optimization procedure.

The estimated values of the meta-parameters and SCV performance during the 10 rounds of DCV and for the SCV on the whole data set are provided in Online Resource 1.

Comparison of subset selection methods

from a statistical perspective

The genes selected by CovProc, LASSO, ELASTIC NET and GA for LEP and TIMP-1 are summarized in Tables 2 and 3, respectively. Genes present in the final SCV models are emboldened. Also shown is the number of occurrences of each gene during the rounds of DCV and the correlation with each protein. The annotations of these genes can be found in Online Resource 2.

CovProc As the predictor data were unit variance scaled, genes are introduced by CovProc based on the magnitude of their correlation with the protein. The final models for LEP and TIMP-1 used the first 16 and 21 most correlated genes, respectively. Note that in both cases, all the selected genes had positive values of $r$ (i.e., positive correlation). For LEP, all the genes in the SCV model were selected at least 9 times during DCV. For TIMP-1, Orm2 was the only gene selected in the SCV model not selected at least 9 times during DCV. Similarly, lfitm 2 was the only gene selected at least 9 times during DCV not to be included in the SCV model. As these are only slight differences, we can conclude that both final models were stable.

LASSO/ELASTIC NET Tables 2 and 3 show that for both proteins, there was considerable consistency between these methods. Both methods used the same genes in their SCV models. The total numbers of genes selected at least once during DCV were also similar, as were the individual genes: there were 21 common genes selected for LEP and 19 for TIMP-1. This can be attributed to the ELASTIC NET models tending toward relatively small values for the ridge parameter and, therefore, behaving similarly to LASSO (see Online Resource 1). For both proteins, all the genes used in the SCV model had significant values of $r$. There was also agreement in the genes selected by these methods and by CovProc. For LEP, all 8 genes were also present in the 16 gene model selected by CovProc. For TIMP-1, there were 5 genes common to the models by LASSO/ELASTIC NET ( 9 genes) and CovProc ( 21 genes) models. This agreement indicates that LASSO and ELASTIC NET preferentially selected genes with high absolute values of $r$. The four genes not present in the TIMP-1 CovProc model were ones less frequently selected during DCV.

GA Models selected by the GA showed the least stability-many genes were selected with a frequency, $f$, of just 1 . In the interests of conciseness, therefore, the results in Tables 2 and 3 comprise genes selected in the final SCV model ordered by the magnitude of the correlation to each protein. For LEP, Mogatl was the most selected during DCV (5 occurrences). For TIMP-1, Serpina $3 n$ was selected in 7 of the DCV models. This was the most correlated gene and was also selected by the other variate selection methods. Of the genes present in the final SCV model, only 7 and 3 were significantly correlated with LEP and TIMP-1, respectively. A total of 281 and 245 genes were selected at least once during DCV for LEP and TIMP-1, respectively, indicating a lack of consistency in the GA models. Two possible contributing factors for this lack of consistency are first, the large model space-10,072 variates-and thus great potential for converging on local minima; and second, that MLR lacks any mechanism for rejecting noise.

Evaluation of variate selection methods

from a biological perspective

To evaluate the biological relevance of the selected subsets and prioritized lists, the following two-step strategy was used. First, a biological function analysis was used to assess whether a given gene list (SCV final model) or gene ranking was biologically meaningful in terms of the significant gene groups they represent. Second, we considered whether these gene groups were consistent with the physiological role of LEP and TIMP-1. 
Table 2 The genes selected by CovProc, LASSO, ELASTIC NET and GA methods for LEP

\begin{tabular}{|c|c|c|c|c|c|c|c|c|c|c|c|}
\hline \multicolumn{3}{|l|}{ CovProc } & \multicolumn{3}{|l|}{ LASSO } & \multicolumn{3}{|l|}{ ELASTIC NET } & \multicolumn{3}{|l|}{ GA } \\
\hline Gene & $f$ & $r$ & Gene & $f$ & $r$ & Gene & $f$ & $r$ & Gene & $f$ & $r$ \\
\hline Cfd & 10 & 0.829 & G0s2 & 10 & 0.826 & G0s2 & 10 & 0.826 & G0s2 & 2 & 0.826 \\
\hline G0s2 & 10 & 0.826 & Cfd & 9 & 0.829 & Cfd & 9 & 0.829 & Mogat1 & 5 & 0.816 \\
\hline Mogat1 & 10 & 0.816 & Mogat1 & 9 & 0.816 & Mogat1 & 9 & 0.816 & D630002G06Rik & 2 & 0.669 \\
\hline Omd & 10 & 0.799 & Cidec & 9 & 0.770 & Cidec & 8 & 0.770 & Elovl5 & 0 & 0.604 \\
\hline Cidea & 10 & 0.797 & Mme & 8 & 0.686 & Mme & 8 & 0.686 & Gstz1 & 0 & 0.582 \\
\hline Clstn3 & 10 & 0.797 & Gpr98 & 7 & 0.755 & Gpr98 & 7 & 0.755 & Apoa4 & 0 & 0.565 \\
\hline Aldh3a2 & 10 & 0.784 & Scd1 & 7 & 0.740 & Scd1 & 7 & 0.740 & Perp & 0 & 0.484 \\
\hline Cidec & 10 & 0.770 & Gstk1 & 5 & 0.755 & Gstk1 & 5 & 0.755 & Bloc1s1 & 0 & 0.266 \\
\hline Gpr98 & 10 & 0.755 & Fabp2 & 4 & 0.683 & Fabp2 & 4 & 0.683 & 2700050L05Rik & 0 & -0.246 \\
\hline Gstk1 & 9 & 0.755 & D630002G06Rik & 4 & 0.669 & D630002G06Rik & 4 & 0.669 & Ubxd1 & 0 & -0.242 \\
\hline Inhbe & 9 & 0.751 & Omd & 3 & 0.799 & Omd & 3 & 0.799 & Ascc3l1 & 0 & -0.222 \\
\hline Tnfrsf19 & 9 & 0.744 & Pgrmc2 & 3 & 0.644 & 3110032G18Rik & 3 & 0.725 & Eif2a & 0 & -0.213 \\
\hline Scd1 & 9 & 0.740 & 3110032G18Rik & 2 & 0.725 & Pgrmc2 & 3 & 0.644 & Lrrc8d & 0 & 0.212 \\
\hline Gpc1 & 9 & 0.733 & Cidea & 1 & 0.797 & $\mathrm{Cd} 36$ & 2 & 0.727 & Pir & 0 & -0.197 \\
\hline Cd36 & 9 & 0.727 & Clstn3 & 1 & 0.797 & Clstn3 & 1 & 0.797 & D2hgdh & 0 & 0.175 \\
\hline 3110032G18Rik & 9 & 0.725 & Gpc1 & 1 & 0.733 & Aldh3a2 & 1 & 0.784 & 9430028L06Rik & 0 & 0.142 \\
\hline S3-12 & 4 & 0.705 & $\mathrm{Cd} 36$ & 1 & 0.727 & Gpc1 & 1 & 0.733 & Myh9 & 0 & -0.121 \\
\hline Apom & 4 & -0.696 & EG624219 & 1 & 0.593 & EG624219 & 1 & 0.593 & Zbtb43 & 0 & 0.116 \\
\hline 1110028A07Rik & 3 & 0.702 & Hectd2 & 1 & 0.567 & Hectd2 & 1 & 0.567 & 0610037D15Rik & 1 & -0.102 \\
\hline Aqp8 & 3 & 0.699 & Nnt & 1 & 0.531 & Apoa4 & 1 & 0.565 & Thnsl2 & 0 & -0.096 \\
\hline Gbe1 & 3 & 0.691 & Abcg5 & 1 & 0.521 & Nnt & 1 & 0.531 & Pitpnm2 & 0 & -0.085 \\
\hline Mme & 3 & 0.686 & Apoc2 & 1 & 0.456 & Abcg5 & 1 & 0.521 & Nt5e & 0 & 0.066 \\
\hline Sema5b & 3 & 0.679 & Aldh3a2 & 0 & 0.784 & Apoc2 & 1 & 0.456 & Il13ra1 & 0 & -0.059 \\
\hline D630002G06Rik & 3 & 0.669 & Inhbe & 0 & 0.751 & Cidea & 0 & 0.797 & Bag2 & 0 & -0.050 \\
\hline Cyp2b9 & 3 & 0.659 & Tnfrsf19 & 0 & 0.744 & Inhbe & 0 & 0.751 & Pik3r4 & 0 & 0.046 \\
\hline Fabp2 & 2 & 0.683 & S3-12 & 0 & 0.705 & Tnfrsf19 & 0 & 0.744 & Xrce6 & 0 & -0.040 \\
\hline Vnn1 & 2 & 0.679 & 1110028A07Rik & 0 & 0.702 & S3-12 & 0 & 0.705 & Sec61a2 & 0 & -0.012 \\
\hline Cryz & 2 & 0.677 & Aqp8 & 0 & 0.699 & 1110028A07Rik & 0 & 0.702 & Tbcc & 0 & -0.006 \\
\hline 16 & 37 & & 8 & 22 & & 8 & 23 & & 28 & 281 & \\
\hline
\end{tabular}

$f$ Number of occurrences in double cross-validation, $r$ correlation coefficient of gene expression and LEP data. Genes present in the final single cross-validation model are in bold. The last row gives the number of genes in the final SCV model (bold) and the number selected at least once during DCV

Overrepresentation of biological functions for CovProc, LASSO, ELASTIC NET and GA selected genes

The biological relevance of the genes selected by the CovProc, LASSO/ELASTIC NET and GA was assessed using biological function overrepresentation analysis within the Ingenuity Pathway Analysis suite. The results are provided in the Online Resource 3. Based on the $p$ value of the biological function category, CovProc performed best with lowest $p$ values of $2.43 \mathrm{E}-06$ and $2.33 \mathrm{E}-$ 07 for LEP and TIMP-1, respectively. LASSO and ELASTIC NET performed similarly with the lowest $p$ value of 5.05E-04 for LEP and 1.44E-05 for TIMP-1. GA performed least well, with a lowest $p$ value of $1.30 \mathrm{E}-$ 03 for LEP and 1.15E-03 for TIMP-1. These results are in broad agreement with the regression-based evaluation of these methods.

Gene Set Enrichment Analysis of $r$ - and PLS-ranked gene lists

GSEA of LEP found 40 (22 positively and 18 negatively) significantly enriched gene sets using correlation $r$-based ranking, and 3 (3 positively and 0 negatively) using PLS regression vector stability ranking. For TIMP-1, GSEA found 51 (29 positively and 22 negatively) and 33 (16 positively and 17 negatively) enriched gene sets using the $r$ - and PLS-ranked lists, respectively.

Investigation into the overlaps between gene sets identified by the two ranking approaches found that all (LEP) or 
Table 3 The genes selected by CovProc, LASSO, ELASTIC NET and GA methods for TIMP-1

\begin{tabular}{|c|c|c|c|c|c|c|c|c|c|c|c|}
\hline \multicolumn{3}{|l|}{ CovProc } & \multicolumn{3}{|l|}{ LASSO } & \multicolumn{3}{|l|}{ ELASTIC NET } & \multicolumn{3}{|l|}{ GA } \\
\hline Gene & $f$ & $r$ & Gene & $f$ & $r$ & Gene & $f$ & $r$ & Gene & $f$ & $r$ \\
\hline Serpina3n & 10 & 0.862 & Serpina3n & 10 & 0.862 & Serpina3n & 9 & 0.862 & Serpina3n & 7 & 0.862 \\
\hline Len2 & 10 & 0.839 & Apcs & 8 & 0.807 & Apcs & 7 & 0.807 & Antxr2 & 0 & 0.581 \\
\hline Serpina10 & 10 & 0.839 & Cobl & 8 & 0.781 & Cpb2 & 7 & 0.784 & Keg1 & 0 & -0.524 \\
\hline Saa2 & 10 & 0.828 & B3galt1 & 8 & 0.780 & Cobl & 7 & 0.781 & Cul1 & 0 & 0.462 \\
\hline Fgl1 & 10 & 0.827 & Cpb2 & 6 & 0.784 & B3galt1 & 6 & 0.780 & Pscdbp & 0 & 0.414 \\
\hline Itih4 & 10 & 0.821 & Hapln4 & 5 & 0.709 & Hapln4 & 3 & 0.709 & Ugcgl1 & 0 & 0.401 \\
\hline Lbp & 10 & 0.820 & Cyb561 & 3 & 0.819 & Spp1 & 3 & 0.631 & LOC634731 & 0 & 0.395 \\
\hline Cyb561 & 10 & 0.819 & Cxcl1 & 3 & 0.686 & Arl6ip5 & 3 & 0.629 & Ica1 & 0 & 0.367 \\
\hline Cpne8 & 10 & 0.813 & Gm527 & 3 & 0.653 & Cxcl1 & 2 & 0.686 & Pilra & 1 & 0.364 \\
\hline Apcs & 10 & 0.807 & 2200001I15Rik & 3 & 0.640 & Gm527 & 2 & 0.653 & Nudt18 & 0 & 0.338 \\
\hline Tnfrsf1a & 10 & 0.805 & Spp1 & 3 & 0.631 & 2200001I15Rik & 2 & 0.640 & Unk & 1 & 0.329 \\
\hline Slc41a2 & 9 & 0.813 & Arl6ip5 & 3 & 0.629 & Serpina10 & 1 & 0.839 & Cyp17a1 & 0 & 0.299 \\
\hline Itih3 & 9 & 0.790 & Fgl1 & 2 & 0.827 & Cyb561 & 1 & 0.819 & Pdia6 & 0 & 0.296 \\
\hline Tmem176a & 9 & 0.785 & Acp6 & 2 & -0.554 & Tnfrsf1a & 1 & 0.805 & Rap2b & 0 & 0.248 \\
\hline Stat3 & 9 & 0.784 & Serpina10 & 1 & 0.839 & Tmem176a & 1 & 0.785 & Pqlc2 & 0 & 0.236 \\
\hline Cpb2 & 9 & 0.784 & Itih4 & 1 & 0.821 & $\mathrm{Mt} 2$ & 1 & 0.784 & Tlr8 & 0 & 0.196 \\
\hline Mt2 & 9 & 0.784 & Tnfrsf1a & 1 & 0.805 & Abhd14b & 1 & -0.693 & Tbc1d13 & 0 & -0.176 \\
\hline Lrg1 & 9 & 0.783 & Tmem176a & 1 & 0.785 & Polg2 & 1 & -0.630 & Cxxc5 & 0 & -0.145 \\
\hline Cobl & 9 & 0.781 & Litaf & 1 & 0.760 & Edg5 & 1 & 0.585 & 1700006J14Rik & 0 & -0.145 \\
\hline B3galt1 & 9 & 0.780 & Abhd14b & 1 & -0.693 & Acp6 & 1 & -0.554 & NA & 0 & 0.132 \\
\hline Ifitm2 & 9 & 0.776 & Polg2 & 1 & -0.630 & $\operatorname{Lcn} 2$ & 0 & 0.839 & Itfg1 & 0 & 0.131 \\
\hline Orm2 & 8 & 0.790 & Edg5 & 1 & 0.585 & Saa2 & 0 & 0.828 & Fstl1 & 0 & 0.098 \\
\hline Zbp1 & 8 & 0.766 & $\operatorname{Lcn} 2$ & 0 & 0.839 & Fgl1 & 0 & 0.827 & AI854517 & 0 & 0.081 \\
\hline Orm1 & 8 & 0.763 & Saa2 & 0 & 0.828 & Itih4 & 0 & 0.821 & Ccdc79 & 0 & -0.036 \\
\hline Litaf & 8 & 0.760 & Lbp & 0 & 0.820 & Lbp & 0 & 0.820 & 5730410I19Rik & 0 & -0.029 \\
\hline Hp & 7 & 0.765 & Cpne8 & 0 & 0.813 & Cpne8 & 0 & 0.813 & Dync2li1 & 1 & 0.015 \\
\hline 21 & 148 & & 9 & 22 & & 9 & 20 & & 26 & 245 & \\
\hline
\end{tabular}

$f$ Number of occurrences in double cross-validation, $r$ correlation coefficient of gene with TIMP-1 data. Genes present in the final single crossvalidation model are in bold. The last row gives the number of genes in the final SCV model (bold) and the number selected at least once during DCV

most (TIMP-1) of the gene sets identified using PLS ranking were also identified by the $r$-based approach. This was true for both positively and negatively enriched gene sets (Fig. 1a, b). Only two positively enriched gene sets found using PLS ranking for TIMP-1 were not also found using the $r$-based approach.

Interestingly, many gene sets that were found positively enriched in the LEP GSEA results were also negatively enriched in the TIMP-1 results and vice versa (Table 4). This is likely a consequence of the biological roles of these two proteins. TIMP-1 and LEP are associated with inflammation and fat metabolism, respectively, processes perturbed during hepatic response to a high-fat challenge. These responses are conversely timed: inflammation is evoked during the early phase (day 1 to week 2 ) and repressed during the late phase (week 4 to week 16) of the high-fat diet response, while lipid metabolic adaptations

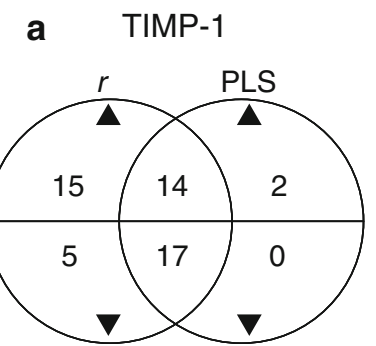

b LEP

Fig. 1 Venn diagrams comparing the numbers of significantly enriched gene sets from GSEA using $r$ - and PLS-based ranking for a TIMP-1 and b LEP. The arrow direction depicts whether the comparison concerns numbers of gene sets with positive (filled triangle) or negative (filled inverted triangle) enrichment

show an opposite temporal pattern and are repressed during early and induced during the late phase of the high-fat feeding time-course (Radonjic et al. 2009). Given the 
inverse temporal expression of LEP and TIMP-1 under the experimental conditions used in this study (data not shown), it may be expected that gene sets that are positively correlated with the expression of the one protein are negatively correlated with the expression of the other protein.

Relevance of biological analysis results in the context of LEP and TIMP-1 functions

Measurements of plasma protein concentrations of Leptin (LEP) and Tissue inhibitor of metalloproteinase 1 (TIMP-1) were considered as two dependent variates for the analysis in this study. These proteins were chosen due to their relevance in addressing the following research question: What are the processes underlying onset and progression of metabolic disorders (such as obesity) associated with high-fat feeding? The early hepatic effect of high-fat feeding involves induction of inflammatory and immune processes, while the late adaptation to excess dietary fat results in hepatic fat accumulation and development of hepatic steatosis (Radonjic et al. 2009). A statistically significant association between circulating plasma parameters and these hepatic physiological processes may be employed for the development of noninvasive diagnostics of the systemic disorder caused by high-fat feeding. To specifically target the representatives of inflammatory and adipogenic processes, we selected TIMP-1 and LEP plasma protein levels from the pool of plasma parameters that were assessed in the high-fat feeding study (Radonjic et al. 2009).

LEP is a circulating adipocytokine that regulates fat mass in response to nutritional status. It plays an important role in maintaining energy homeostasis and metabolic rate and its plasma levels are affected by energy-rich nutrients such as fatty acids, carbohydrates and proteins (Ahima and Flier 2000; Zou and Shao 2008). In agreement with the physiological role of LEP, the most significant functional category identified by the analysis of genes in the CovProc final SCV model is related to lipid metabolism (Online Resource 3). Also with high significance ( $p=4.75 \mathrm{E}-04)$, was the category "carbohydrate metabolism." Lipid and carbohydrate metabolism were also represented in LASSO/ ELASTIC NET $(p=3.03 \mathrm{E}-03)$ and GA results ( $p=1.30 \mathrm{E}-03$ to $7.77 \mathrm{E}-03$ ). Additionally, the GA model identified genes involved in metabolism of amino acids/ proteins. Consistent with the role of LEP, GSEA found significant positively enriched gene sets related to amino acid metabolism, fatty acid metabolism, energy yielding processes such as oxidative phosphorylation and tricarboxylic acid (TCA) cycle, and conditions associated with increased adiposity (Table 4). In the context of using subset selection methods (CovProc, LASSO/ELASTIC
NET and GA) to find markers associated with a given biological parameter, GOs2, Cfd and Mogatl could be considered as the top three markers associated with LEP. They were selected by all the final models, and all have functions associated with lipid metabolism. Specifically, GOS2 regulates adipose lipolysis; CFD (adipsin) is involved in systemic lipid metabolism or energy balance; and MOGAT1 catalyzes the synthesis of precursors of physiologically important lipids such as triacylglycerol and phospholipids (Cook et al. 1987, Yen et al. 2003, Yang et al. 2010). Regarding the crucial role of LEP in energy homeostasis, lipid metabolism and liver pathophysiology, the specific processes mediated by GOS2, CFD and MOGAT1 may suggest the possible routes via which LEP accomplishes these functions.

TIMP-1 has a role in the degradation of extracellular matrix proteins in response to various stimuli in both normal and pathological conditions including morphogenesis, tissue repair, tumorigenesis and cell death (Gaudin et al. 2000; Guedez et al. 1996; Ray and Stetler-Stevenson 1994). Additionally, TIMP-1 is produced by lymphocytes as an important factor in facilitating leukocyte infiltration into inflammatory sites during inflammatory response (Johnatty et al. 1997). In agreement with the roles of TIMP-1, the most significant functional category identified by the CovProc SCV final model is related to "inflammatory response" ( $p=2.33 \mathrm{E}-07$ ) (see Online Resource 3). The category "Hepatic System Disease" is also found significant among CovProc results (1.75E-04). Similarly, the category "inflammatory response" is also highly significant among LASSO and ELASTIC NET results $(p=1.44 \mathrm{E}-05)$. The GA method performed less well, with $p$ value of $1.72 \mathrm{E}-02$ for the same category. The significant positively enriched gene sets identified by GSEA of TIMP-1 are associated with several pathological states, including inflammation-related pathologies, tissue rejection during transplantation, hepatomas, hepatitis and disorders caused by inflammatory agents (Table 4). The overlap of significant gene sets with Gene Ontology categories (The Gene Ontology Consortium 2000) reveals that "immune system process" and "inflammatory response" are the most relevant biological processes underlying the above listed pathologies ( $p$ value $4.58 \mathrm{E}-9$ and $2.82 \mathrm{E}-7$, respectively, for the significance of the overlap with the most significant gene set). For TIMP-1, Serpina3n was selected as the top-ranked associated gene in all the final models (CovProc, LASSO/ELASTIC NET and GA) and can, therefore, be considered as the most relevant marker. SERPINA3N is a protease inhibitor, and deficiency of this protein has been linked to liver disease. A direct functional link between TIMP-1 and SERPINA3N has not been established yet, but from their cellular roles, it is likely that they act interdependently in degrading the extracellular 
Table 4 Enriched gene sets identified by GSEA using $r$-based ranking

\begin{tabular}{|c|c|}
\hline \multicolumn{2}{|l|}{ Gene sets } \\
\hline ABBUD_LIF_UP (T5) [47] & $\Delta$ \\
\hline ADIP_VS_PREADIP_DN & $\Delta$ \\
\hline AGEING_KIDNEY_SPECIFIC_UP & $\Delta$ \\
\hline BASSO_GERMINAL_CENTER_CD40_UP & $\boldsymbol{\Delta}$ \\
\hline BROCKE_IL6 & $\Delta \mathbf{0}$ \\
\hline DAVIES_MGUS_MM & $\Delta$ \\
\hline IDX_TSA_DN_CLUSTER1 & $\boldsymbol{\Delta}$ \\
\hline KRETZSCHMAR_IL6_DIFF & $\Delta \mathbf{0}$ \\
\hline LEE_DENA_UP (T2) [60] & $\boldsymbol{\Delta}$ \\
\hline LEE_MYC_E2F1_UP & $\boldsymbol{\Delta}$ \\
\hline LIAN_MYELOID_DIFF_GRANULE & $\Delta$ \\
\hline LIAN_MYELOID_DIFF_RECEPTORS & $\Delta$ \\
\hline LINDSTEDT_DEND_8H_VS_48H_UP & $\boldsymbol{\Delta}$ \\
\hline NI2_MOUSE_UP (T2) [40] & $\Delta$ \\
\hline RIBAVIRIN_RSV_UP & $\Delta$ \\
\hline ROSS_CBF_MYH & $\boldsymbol{\Delta}$ \\
\hline TAKEDA_NUP8_HOXA9_3D_UP & $\boldsymbol{\Delta}$ \\
\hline AGEING_KIDNEY_UP & $\mathbf{\Delta} \nabla$ \\
\hline CARIES_PULP_HIGH_UP & $\mathbf{\Delta} \nabla \mathbf{O}$ \\
\hline CARIES_PULP_UP & $\mathbf{\Delta} \nabla \mathbf{O}$ \\
\hline $\begin{array}{l}\text { FLECHNER_KIDNEY_TRANSPLANT_ } \\
\text { REJECTION_UP }\end{array}$ & $\mathbf{\Delta} \nabla \bullet$ \\
\hline GALINDO_ACT_UP & $\mathbf{\Delta} \nabla$ \\
\hline ICHIBA_GVHD (T6) [335] & $\mathbf{\Delta} \nabla \mathbf{O}$ \\
\hline LAL_KO_3MO_UP & $\mathbf{\Delta} \nabla \mathbf{O}$ \\
\hline LAL_KO_6MO_UP & $\mathbf{\Delta} \nabla \mathbf{O}$ \\
\hline NADLER_OBESITY_UP & $\mathbf{\Delta} \nabla \mathbf{O}$ \\
\hline NEMETH_TNF_UP & $\mathbf{\Delta} \nabla$ \\
\hline TARTE_PC & $\mathbf{\Delta} \nabla \mathbf{0}$ \\
\hline WIELAND_HEPATITIS_B_INDUCED & $\mathbf{\Delta} \nabla$ \\
\hline AGEING_KIDNEY_SPECIFIC_DN & $\nabla \triangle \bullet$ \\
\hline BETA_ALANINE_METABOLISM & $\nabla \triangle 0$ \\
\hline BUTANOATE_METABOLISM & $\boldsymbol{\nabla} \triangle \bigcirc$ \\
\hline ELECTRON_TRANSPORT_CHAIN & $\nabla \triangle \bullet$ \\
\hline FATTY_ACID_DEGRADATION & $\nabla \triangle$ \\
\hline FATTY_ACID_METABOLISM (L2) [86] & $\nabla \triangle \bullet$ \\
\hline $\begin{array}{l}\text { FLECHNER_KIDNEY_TRANSPLANT_ } \\
\text { REJECTION_DN }\end{array}$ & $\nabla \triangle \bullet$ \\
\hline HUMAN_MITODB_6_2002 & $\nabla \triangle$ \\
\hline IDX_TSA_UP_CLUSTER6 (L2) [166] & $\nabla \triangle 0$ \\
\hline KREBS_TCA_CYCLE & $\boldsymbol{\nabla} \triangle \bigcirc$ \\
\hline LEE_DENA_DN & $\nabla \triangle \boldsymbol{O}$ \\
\hline LYSINE_DEGRADATION & $\nabla \triangle$ \\
\hline MITOCHONDRIA & $\nabla \triangle 0$ \\
\hline MOOTHA_VOXPHOS & $\nabla \triangle 0$ \\
\hline PROPANOATE_METABOLISM & $\nabla \triangle 0$ \\
\hline $\begin{array}{l}\text { VALINE_LEUCINE_AND_ISOLEUCINE_ } \\
\text { DEGRADATION }\end{array}$ & $\boldsymbol{\nabla} \triangle \bigcirc$ \\
\hline
\end{tabular}

Table 4 continued

\begin{tabular}{lc}
\hline Gene sets & \\
\hline BILE_ACID_BIOSYNTHESIS & $\boldsymbol{\nabla}$ \\
GLUTATHIONE_METABOLISM & $\boldsymbol{\nabla}$ \\
HCC_SURVIVAL_GOOD_VS_POOR_UP & $\boldsymbol{\nabla}$ \\
IDX_TSA_UP_CLUSTER5 & $\boldsymbol{\nabla}$ \\
TRYPTOPHAN_METABOLISM & $\boldsymbol{\nabla}$ \\
WANG_MLL_CBP_VS_GMP_DN & $\boldsymbol{\nabla}$ \\
ADIP_VS_FIBRO_UP (L3) [35] & $\triangle$ \\
ADIP_VS_PREADIP_UP (L3) [36] & $\triangle$ \\
LEE_CIP_UP (L4) [62] & $\triangle$ \\
LEE_MYC_TGFA_DN & $\triangle$ \\
TNFALPHA_ADIP_DN (L2) [59] & $\triangle$ \\
ZMPSTE24_KO_DN (L2) [32] & $\triangle$ \\
IDX_TSA_DN_CLUSTER2 & $\nabla$ \\
MYOD_NIH3T3_DN & $\nabla$ \\
ROS_MOUSE_AORTA_DN & $\nabla$ \\
STEMCELL_COMMON_DN & $\nabla$ \\
TRANSLATION_FACTORS & $\nabla$ \\
TRNA_SYNTHETASES & $\nabla$
\end{tabular}

Key to symbols positively $(\boldsymbol{\Delta})$ and negatively $(\boldsymbol{\nabla})$ enriched gene sets found for TIMP-1; positively $(\triangle)$ and negatively $(\nabla)$ enriched gene sets found for LEP; gene sets also found using PLS-based ranking for TIMP-1( $)$ and LEP $(\bigcirc)$. Emboldened gene sets were also identified from the CovProc selected variates. The size of the gene set is given in square brackets, and the number of CovProc identified genes present for TIMP-1 (T) or LEP (L) is shown in round brackets

matrix proteins during inflammatory response and/or other conditions.

Considering the functions of LEP and TIMP-1, we may conclude that all methods performed well in the identification of biologically relevant genes.

In summary, CovProc was the best performing MVA subset selection method. Similarly, for GSEA, the $r$-based ranking performed better than the ranking based on the stability of the PLS regression coefficients. In terms of biological relevance, the choice between these two methods will depend on the research goal. While CovProc will be more suitable for selecting a limited set of markers associated with a given dependent parameter, GSEA using $r$-based ranking may provide a more global insight into biological processes related to this parameter.

Direct comparison of CovProc selected variates

with pathways prioritized by the ranking methods

To directly compare CovProc selected variates with pathways prioritized by the ranking methods, the 16 and 21 genes used in the final SCV CovProc models for LEP and TIMP-1, respectively (bold in Tables 2 and 3), were overlapped with the total $\mathrm{C} 2$ gene sets collection $(1,892$ 
gene sets including 17,544 genes). Using a $p$ value threshold of 0.01, 15 gene sets were identified for LEP and 9 for TIMP-1.

Of the identified gene sets, 7 and 4 were also identified by $r$-ranked GSEA and 0 and 3 identified by PLS-ranked GSEA for LEP and TIMP-1, respectively (Table 4). This shows that the biological interpretation of genes selected by CovProc corresponds well with the interpretation of the $r$-ranked results. All the overlapping gene sets between $r$ and CovProc are found among positively enriched gene sets. This is consistent with CovProc selected genes that were exclusively positively correlated with LEP and TIMP-1.

\section{Conclusions}

This study has compared five methods currently used for variate selection or ranking: PLS, GA, LASSO/ELASTIC NET and CovProc. Based on statistical model performance and parsimony, the GA is outperformed by the other methods, with CovProc as the best method. From a biological perspective, it appears that all methods select meaningful variates, either for variate subsets (CovProc, LASSO/ELASTIC NET) or for gene rankings (correlation and PLS coefficient stability), although CovProc somewhat outperforms the other methods for selecting a definite list of genes. We would also recommend that any multivariate analysis should be used in conjunction with more traditional univariate analyses. The results of biological interpretation using $r$-based rankings are superior to those using ranking by PLS coefficient stability.

In conclusion, based on the biological interpretability of the results, CovProc and correlation-ranked methods are both highly recommended, complementary methods for analyzing transcriptomic data. CovProc is particularly suited to select a limited set of markers associated with a given biological parameter, while correlation-ranked GSEA is more appropriate for gaining global insight into biological processes associated with that parameter.

Acknowledgments Age Smilde (Universiteit van Amsterdam, The Netherlands) and Marjan van Erk (TNO, The Netherlands) are thanked for their discussions. This work has been performed with the help of a grant of The European Nutrigenomics Organisation linking genomics, nutrition and health research (NuGO). HST was funded by the Biotechnology \& Biological Sciences Research Council under their Strategic Competitive Grant. MR and UT were funded by Top Institute Food and Nutrition, The Netherlands (Nutrigenomics Consortium, project A-006 (http://www.nutrigenomicsconsortium.nl).

Open Access This article is distributed under the terms of the Creative Commons Attribution License which permits any use, distribution, and reproduction in any medium, provided the original author(s) and the source are credited.

\section{References}

Afman L, Müller M (2006) Nutrigenomics: from molecular nutrition to prevention of disease. J Am Diet Assoc 106:569-576

Ahima RS, Flier JS (2000) Adipose tissue as an endocrine organ. Trends Endocrinol Metab 11:327-332

Baccini M et al (2008) The NuGO proof of principle study package: a collaborative research effort of the European Nutrigenomics Organisation. Genes Nutr 3:147-151

Benjamini Y, Hochberg Y (1995) Controlling the false discovery rate: a practical and powerful approach to multiple testing. J R Stat Soc Ser B 57(1):289-300

Boulesteix AL, Strimmer K (2007) Partial least squares: a versatile tool for the analysis of high-dimensional genomic data. Brief Bioinform 8:32-44

Cook KS et al (1987) Adipsin: a circulating serine protease homolog secreted by adipose tissue and sciatic nerve. Science 237:402405

Evelo CT et al (2011) Answering biological question-querying a systems biology database for nutrigenomics. Genes Nutr 6:81-87

Faber NM (2002) Uncertainty estimation for multivariate regression coefficients. Chemom Intell Lab Syst 64:169-179

Gaudin P et al (2000) TIMP-1/MMP-9 imbalance in an EBVimmortalized B lym-phocyte cellular model: evidence for TIMP1 multifunctional properties. Biochim Biophys Acta-Mol Cell Res 11:19-33

Guedez L et al (1996) The role of metalloproteinases and their inhibitors in hematological disorders. Crit Rev Oncog 7:205-225

Guyon I, Elisseeff A (2003) An introduction to variable and feature selection. J Mach Learn Res 3:1157-1182

Guyon I, Gunn S, Nikravesh M, Zadeh L (eds) (2006) Feature extraction, foundations and applications. Series Studies in Fuzziness and Soft Computing. Physica-Verlag, Springer, Berlin

Johnatty RN et al (1997) Cytokine and chemokine regulation of proMMP-9 and TIMP-1 production by human peripheral blood lymphocytes. J Immunol 158:2327-2333

Kaput J et al (2010) Connecting the Human Variome Project to nutrigenomics. Genes Nutr 5:275-283

Kemsley EK et al (2007) Multivariate techniques and their application in nutrition: a metabolomics case study. Br J Nutr 98:1-14

Martens H, Naes T (1989) Multivariate calibration, 2nd edn. Wiley, Chichester

McLeod G et al (2009) A comparison of variate pre-selection methods for use in partial least squares regression: a case study on NIR spectroscopy applied to monitoring beer fermentation. J Food Eng 90:300-307

Mitchelle M (1998) An Introduction to Genetic Algorithms. MIT Press, Cambridge, MA

Müller M, Kersten S (2003) Nutrigenomics: goals and perspectives. Nat Rev Genet 4:315-322

Norušis MJ, SPSS Inc (1990) SPSS base system user's guide. SPSS, SPSS Inc, Chicago

Radonjic M et al (2009) Genome-wide mRNA expression analysis of hepatic adaptation to high-fat diets reveals switch from an inflammatory to steatotic transcriptional program. PLoS ONE 4:e6646

Ray JM, Stetler-Stevenson WG (1994) The role of matrix metalloproteases and their inhibitors in tumour invasion, metastasis and angiogenesis. Eur Respir J 7:2062-2072

Reinikainen S-P, Höskuldsson A (2003) COVPROC method: strategy in modeling dynamic systems. J Chemom 17:130-139 
Smit S et al (2007) Assessing the statistical validity of proteomics based biomarkers. Anal Chim Acta 592:210-217

Stone M (1974) Cross-validatory choice and assessment of statistical predictions. J R Stat Soc B 36:111-147

Subramanian A et al (2005) Gene set enrichment analysis: a knowledge-based approach for interpreting genome-wide expression profiles. Proc Natl Acad Sci 102:15545-15550

The Gene Ontology Consortium (2000) Gene Ontology: tool for the unification of biology. Nat Genet 25:25-29

Tibshirani R (1996) Regression shrinkage and selection via the lasso. J R Stat Soc B 58:267-288

Van den Berg RA (2006) Centering, scaling, and transformations: improving the biological information content of metabolomics data. BMC Genomics 7:142
Yang $\mathrm{X}$ et al (2010) The G0/G1 switch gene 2 regulates adipose lipolysis through association with adipose triglyceride lipase. Cell Metab 11:194-205

Yen CL et al (2003) MGAT2, a monoacylglycerol acyltransferase expressed in the small intestine. J Biol Chem 278:18532-18537

Zou H, Hastie T (2005) Regularisation and variable selection via the elastic net. J R Stat Soc B 67:301-320

Zou C, Shao J (2008) Role of adipocytokines in obesity-associated insulin resistance. J Nutr Biochem 19:277-286 